\title{
Optimización de lubricantes de base mineral de motores a inyección de gasolina, para la reducción de la contaminación y costos de mantenimiento
}

\author{
Luis Fernando Macas Redrován, Miriam Mancheno, \\ Diego Fernando Jaramillo Sari y Diego René Urgilés Contreras \\ lredrovan@est.ups.edu.ec
}

\section{Resumen}

La aplicación de un mantenimiento preventivo y control técnico incide directamente en el rendimiento del lubricante del motor, lo que se traduce en un factor favorable para el medio ambiente y la economía de los usuarios.

La ejecución de este proyecto, ejecutado en la ciudad de Cuenca, requirió se realice una gestión con las diversas empresas de taxis para incluir 21 unidades al proceso. Durante el desarrollo de la investigación se realizó un acondicionado técnico de los motores de los vehículos para luego del uso extraer la primera muestra de aceite del motor posterior al recorrido que cada propietario de taxi acostumbra hacer, posteriormente, una segunda muestra a un recorrido extendido en un $100 \%$, estas muestras fueron enviadas a un laboratorio especializado en análisis químico. En las muestras se contemplaron parámetros de salud del lubricante, contaminantes e incluso desgaste del motor; como resultado y gracias a la interpretación de dichos parámetros se estableció que un lubricante de viscosidad $10 \mathrm{~W} 30$ es la verdadera aplicación para los motores a gasolina del año de fabricación 2005 en adelante, y que el mismo puede mantenerse en uso por un periodo de $7.000 \mathrm{~km}$ de recorrido en condiciones severas de operación; esto nos da una proyección de economizar un $\mathbf{5 0}$ \% los costos de mantenimiento de motor y disminuir el $50 \%$ de la contaminación por estos desechos automotrices.

Palabras clave: Mantenimiento preventivo, control técnico, lubricantes, vida útil, disminución de la contaminación, disminución de costos.

\section{Abstract}

The application of preventive maintenance and technical control directly affects the performance of motor oil, resulting in a favorable factor for the environment and the economy of the users.

Within the development of this project was carried out with different management of taxi companies to include 21 units to the project, a technical condition of their engines and then extract the first sample of used motor oil to travel to each taxi owner and usually do then a second sample to an extended run at $100 \%$, and each was sent to a chemical laboratory specializing in chemical analysis, which shows health of the lubricant parameters, contaminants and even engine wear, and thanks to the interpretation of such parameters provides that a $10 \mathrm{~W} 30$ viscosity lubricant is the real application for gasoline engines of model year 2005 onwards, and that it may be in use for a period of $7000 \mathrm{~km}$ of travel in severe operating conditions, giving a projected savings by 50\% the cost of engine maintenance and a decrease of 50\% of this waste automotive pollution.

Keywords: Preventing maintenance, technical control, lubricants, unavailable service, environmental decreasing pollution, cost decreasing

Forma sugerida de citar: Macas Redrován, Luis Fernando; Mancheno, Miriam; Jaramillo Sari, Diego Fernando; Urgilés Contreras, Diego René. 2011. “Optimización de lubricantes de base mineral de motores a inyección de gasolina, par la reducción de la contaminación y costos de mantenimiento". Ingenius No. 5. Enero/Junio. Pp. 73-80. 


\section{Introducción}

El no tener un criterio técnico sobre el mantenimiento preventivo automotriz ocasiona consecuencias muy graves, entre las más importantes está el daño al medioambiente; pues, una mala práctica de mantenimiento en un vehículo genera desechos automotrices sólidos y líquidos. Actualmente, la mayoría de los propietarios de vehículos se han dejado llevar por los procedimientos empíricos de mantenimiento, y los han practicado muy a menudo; entre los procedimientos está el 'cambio de aceite del motor a los $3.500 \mathrm{~km}$ de recorrido, o un poco más”.

Con el fin de demostrar el nivel de contaminación por desechos automotrices, realizaremos el siguiente ejercicio. Tomamos una media de 1 galón de aceite de motor por vehículo; existen 3.532 taxis legalmente constituidos en la ciudad de Cuenca ${ }^{1}$; esto nos indica que cada mes se tendría 3.532 galones de aceite usado, tan solo provocado por los taxis; además, se suman los 3.532 filtros para lubricante que se desechan conjuntamente al cambio de lubricante; a estos valores se suman los vehículos de transporte de carga y de pasajeros, tanto público como privado; se debe considerar que estos tiempos de cambio de lubricante de motor, implican costos de mantenimiento significativos.

Por lo menos se tiene un alivio respecto a los lubricantes de motor usados; pues se sabe que son reutilizados como combustible para motores estacionarios de cementeras y fábricas; pero esta reutilización representa mayores y peligrosas emisiones de gases contaminantes que un combustible normal de acuerdo a datos emitidos por la empresa ETAPA (Empresa de Telecomunicaciones Alcantarillado y Agua Potable de Cuenca) en el año 2010.

Así, surge la necesidad de aprovechar de mejor manera los recursos automotrices para reducir la contaminación ambiental y los costos de mantenimiento de motor de los vehículos, con estos objetivos se presenta la siguiente investigación, en donde se muestra la verdadera vida útil de un lubricante para motor a gasolina, a través de la interpretación del análisis químico realizado.

\section{Materiales y métodos}

Durante el proyecto se realizó una investigación del parque automotor de taxis, en la ciudad de Cuenca, determinando el tipo de aceite usado y su periodicidad en el cambio. De esta manera, se plantea una encuesta con la que se pueda determinar el tipo de aceite más usado en los vehículos de servicio de taxi. El estudio se efectuó en vehículos de transporte público (servicio de taxi), porque están sometidos a periodos de funcionamiento más extensos, y producen desechos automotrices sólidos y líquidos en corto tiempo (3 semanas), considerando costos de mantenimiento de motor mayores con respecto a vehículos particulares.

De acuerdo a los archivos de la Unidad Municipal de Tránsito-UMT, a febrero de 2010, se estableció el número de taxis circulantes en la ciudad de Cuenca y se seleccionaron los vehículos con año de fabricación del 2005 en adelante; debido a que los motores de estos vehículos están aún dentro de su vida útil.

Aplicando la formula estadística del tamaño de la muestra sugerida para estos estudios, se justifica un número de 69 vehículos de una población de 1.302. Previo al inicio del Pro- 
yecto se convocó a 69 vehículos, de los cuales asistieron en un número de 33 vehículos, retirándose por causas ajenas 12 vehículos, se culminó el proyecto con 21 unidades; a pesar de ello esta muestra es significativa para el logro de los objetivos planteados. Estas 21 unidades reunían las condiciones mecánicas comunes como: desgaste de motor normal y un correcto estado de los sistemas auxiliares del motor (lubricación, refrigeración, alimentación, sistema de encendido) al igual que la gestión electrónica; cabe indicar que en este grupo de vehículos se encontraban la mayoría de marcas y modelos del parque automotor de taxis de la ciudad de Cuenca.

Sobre esta muestra y para asegurar la permanencia de los vehículos participantes, se ofrecieron los insumos necesarios: aceites, filtros para el sistema de lubricación, admisión de aire y alimentación de combustible, a cada uno, y en caso de presentarse algún inconveniente por el uso, se realizó el compromiso de asumir los posibles costos.

Previo a la inclusión de los automotores en el proyecto se efectuó un diagnóstico del estado de cada uno de los motores (Figura 1), para hacer efectivas las garantías. A continuación se procede a ejecutar un plan de control en donde se registraba el seguimiento a los automo-

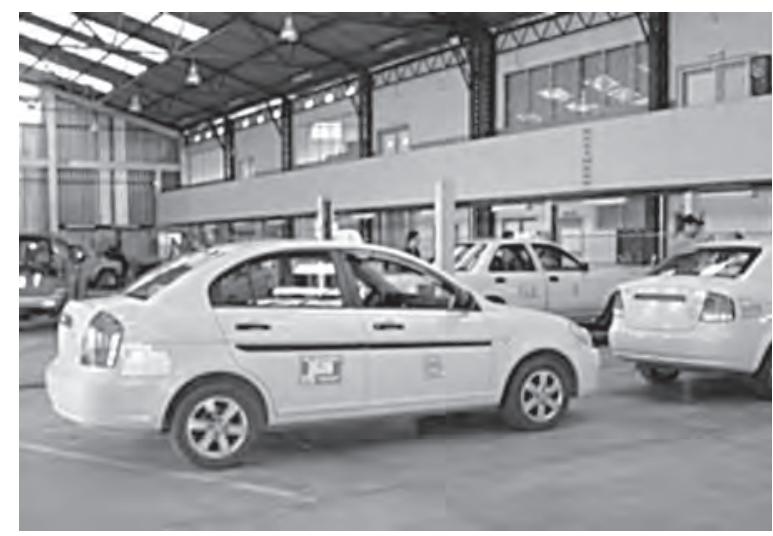

Figura 1. Vehículos participantes

Fuente: Los autores tores, que incluía mantenimientos preventivos, controles técnicos y diagnósticos en el motor de cada vehículo.

Se diseñó un logotipo de identificación del proyecto, el mismo que lo portaron todos los participantes del proyecto.

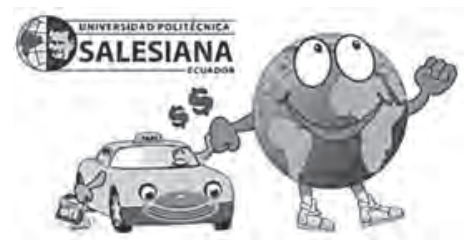

Figura 2. Logotipo de identificación del proyecto. Fuente: Los autores

\subsection{Acondicionamiento de motores}

El acondicionamiento mecánico efectuado en cada automotor implicó realizar mantenimientos basados en procesos técnicos, para restablecer cada vehículo a los parámetros especificados por cada fabricante, y así llevar un seguimiento periódico del motor que permita comprobar su correcto funcionamiento y comportamiento; elaborándose un historial técnico de cada vehículo.

\subsection{Extracción de muestras}

Cada propietario de los automotores estableció un kilometraje de recorrido con base en su experiencia y recomendación, para el cambio de aceite de motor; una vez que cada automotor cumplió con el kilometraje se extrajo la primera muestra de aceite usado para enviarla al laboratorio ANALSYST de la empresa NORIA, ubicada en Monterrey-México, para que se practique su respectivo análisis químico. Seguido a esto, se estableció extender un 100\% los periodos de cambio de aceite para cada vehículo; mientras tanto, durante estos periodos se realizaron controles técnicos puesto que 
éstos experimentaban nuevos kilometrajes de recorrido con el mismo lubricante.

Al cumplimiento de los recorridos extendidos, se procedió a extraer la segunda muestra de aceite usado, y de la misma manera se procedió a enviarla al laboratorio para su respectivo análisis químico.

\subsection{Interpretación de análisis químico}

Al tener los resultados de las muestras de cada vehículo se realizaron las respectivas in- terpretaciones (Analsyst, 2010), que consistieron en determinar la salud del lubricante, analizando el grado de viscosidad del aceite según la norma SAE J300, los números base y ácido, el estado de aditivos; el grado de contaminación existente estableciendo las cantidades de silicio, sodio, potasio, agua y combustible presentes en el lubricante; y, finalmente los metales de desgaste del motor que permitan conocer el nivel de desgaste interno de la máquina (Figura 3); límites determinados según líneas de tendencia de estudios anteriores realizados por entes internacionales.

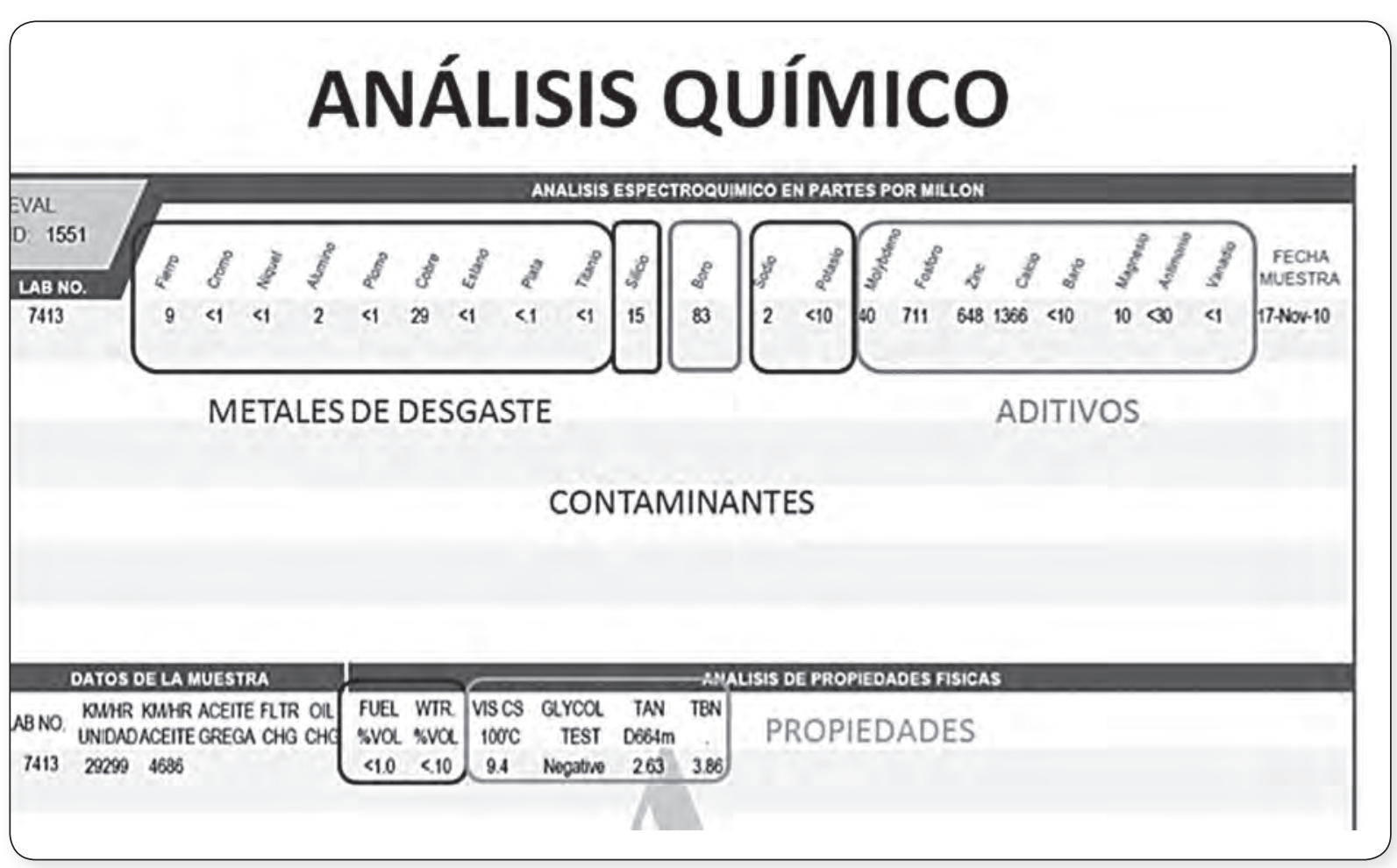

Figura 3. Análisis químico de lubricante de motor

Fuente: Laboratorio ANALSYST 


\section{Resultados}

La vida útil de un lubricante está relacionada directamente con: el diseño del motor ya que existen partes con holguras bien dimensionadas en donde se permite únicamente una película de lubricante entre las piezas y todo ello difiere del tipo de motor; la calidad del lubricante utilizado se nota directamente en la cantidad de aditivos que posee y su TBN (Número Total Base); mientras éstos sean valores altos, el lubricante ofrecerá mayor protección al motor, siempre y cuando se aplique la viscosidad correcta; para la elección del lubricante se debe consultar el manual del fabricante del vehículo; el mantenimiento del motor es el factor más importante puesto que determinará la vida del motor, un buen plan de mantenimiento basado en las recomendaciones del fabricante y orientado a nuestro entorno, permite optimizar la vida útil del lubricante.

\subsection{Viscosidad}

Una vez recopilada la información de los análisis químicos correspondientes se encontraron claras diferencias, las que marcaron la eficiencia de un aceite $20 \mathrm{~W} 50$ con respecto al 10W30, esto se pudo comprobar mediante al análisis de los factores más comunes y críticos entre los dos aceites (Figura 4).

El rango de viscosidad a $100^{\circ} \mathrm{C}$ de un aceite 20W50 es " 16,3 cSt - 21,9cSt", el cual está diseñado para trabajar a temperaturas entre $118^{\circ} \mathrm{C}$ y $130^{\circ} \mathrm{C}$; la Figura 4 indica que la mayoría de los lubricantes caen fuera del rango establecido, disminuyendo su viscosidad por cizallamiento, fenómeno que se da en las zonas de mayor presión y temperatura del motor.

El rango de viscosidad a $100^{\circ} \mathrm{C}$ de un aceite 10W30 es "9,3 cSt - 12,5 cSt", el cual está diseñado para trabajar a temperaturas entre $92^{\circ} \mathrm{C}$ y $107^{\circ} \mathrm{C}$; la Figura 5 muestra que la mayoría de los lubricantes se mantienen en el rango establecido por la Norma SAE J300.

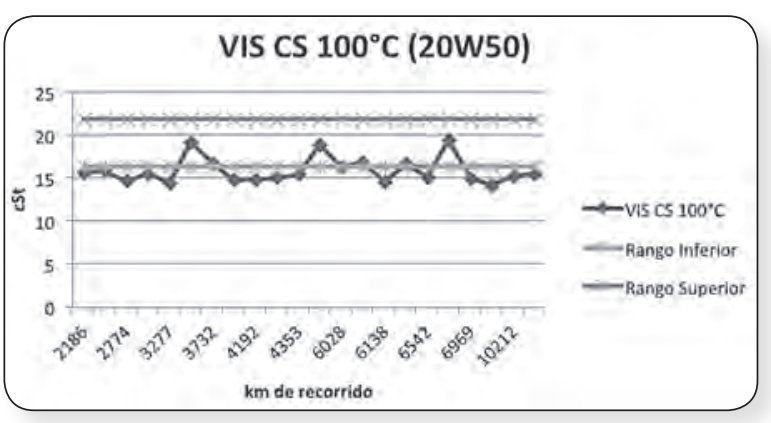

Figura 4. Comportamiento de la viscosidad a $100^{\circ} \mathrm{C}$ en un aceite 20W50

Fuente: Los autores.

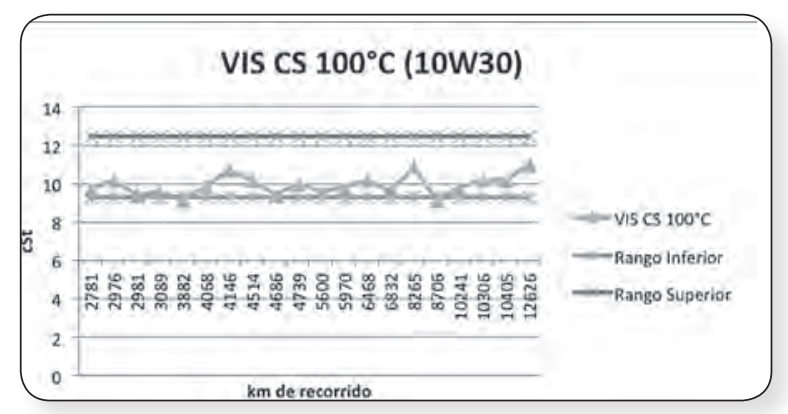

Figura 5. Comportamiento de la viscosidad a $100^{\circ} \mathrm{C}$ en un aceite $10 \mathrm{~W} 30$

Fuente: Los autores.

\subsection{TAN (Número Total Ácido) vs. TBN (Número Total Base)}

De la misma manera que en el punto anterior se establece un análisis con respecto a las características más importantes de un lubricante; en donde se tiene como criterio que la vida útil del mismo llega hasta que éstos números alcancen los mismos valores; por otra parte, un lubricante que puede continuar en uso es cuando el TBN es más alto que el TAN.

La Figura 6 muestra las líneas de tendencia; y según los criterios de vida útil del lubricante 


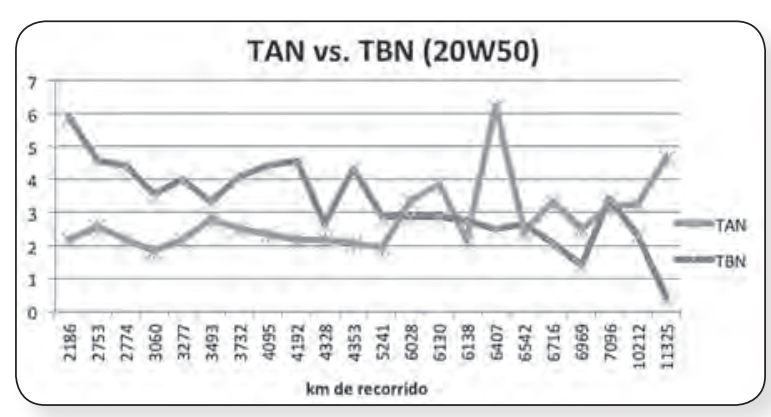

Figura 6. Comportamiento de TAN vs. TBN en un aceite 10W30

Fuente: Los autores.

se tiene que un lubricante 20 W50 puede estar dentro del motor hasta $5.300 \mathrm{~km}$ de recorrido aproximadamente, pues hasta allí los números TAN y TBN son de igual cantidad, y desde éste en adelante el lubricante comienza a convertirse en un agente corrosivo para el motor. A pesar de que este lubricante puede durar este periodo, fijese en el comportamiento de viscosidad de la Figura 5, en lo que se explica que el lubricante no está protegiendo de manera óptima al motor.

Por otra parte, la línea de tendencia de la Figura 7 muestra una durabilidad de $6.700 \mathrm{~km}$ aproximadamente, y con respecto a un lubricante 20W50 se muestra una línea más estable; además se nota una clara diferencia en el comportamiento de viscosidad que se muestra en la Figura 5; demostrando que el lubricante 10W30 está protegiendo al motor durante su vida útil.

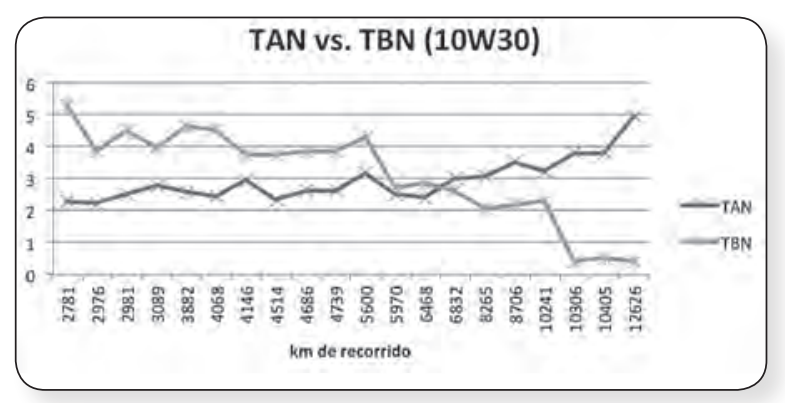

Figura 7. Comportamiento de TAN vs. TBN en un aceite 10W30

Fuente: Los autores.
Cabe destacar que estas líneas de tendencia han sido elaboradas con los resultados de todos los vehículos participantes, que han circulado por la ciudad de Cuenca bajo condiciones de conducción severas.

\subsection{Conducción técnica}

La mayoría de participantes conducen sus vehículos a bajas revoluciones para apegarse al mito de ahorrar combustible; mientras que esto no es así, porque al conducir a bajas revoluciones hay un mayor consumo de combustible, puesto que la gestión electrónica dosifica una mayor cantidad de combustible para evitar que el motor comience a vibrar de manera excesiva, o más conocido como el golpe cilindro-pistón (cascabeleo), fenómeno que acelera el desgaste del motor. Una conducción técnica, implica seguir las especificaciones del fabricante; obteniéndose la máxima potencia, el máximo rendimiento y el mínimo consumo de combustible.

\section{Conclusiones}

El estudio realizado demuestra que gran parte de los propietarios de las unidades de taxis en la ciudad de Cuenca realizan los cambios de aceite de motor basados en conocimientos empíricos, pues son muy pocos los que siguen las recomendaciones del fabricante al aplicar un mantenimiento técnico basado en este tipo de criterios garantiza un adecuado funcionamiento del motor y también una correcta optimización de recursos, especialmente de aceite de motor, el cual es limitado en la mayoría de casos por incorrectos criterios de mantenimiento.

Uno de los factores que afectan directamente la vida del motor y el lubricante es la forma de conducir. En los vehículos estudiados se aprecia que la conducción a bajas revoluciones 
se presenta en muchos de los casos, lo cual provoca fenómenos como golpe cilindro-pistón (cascabeleo) y demasiado esfuerzo del motor, estas pérdidas de potencia son compensadas mediante la gestión electrónica que inyecta mayor cantidad de combustible que contamina el lubricante, reduciendo su vida útil.

Durante el estudio, un 61,9 \% de los vehículos participantes utilizan un aceite de viscosidad 20W50, dicha característica se pierde de forma prematura debido a fenómenos de cizallamiento, esto se da en las zonas de mayores presión y temperatura en el motor, y según las características de diseño del aceite este trabajará en un rango de temperaturas de $118{ }^{\circ} \mathrm{C}$ a $130{ }^{\circ} \mathrm{C}$ para una protección y lubricación óptima, las que no se alcanzan en este tipo de motores. El lubricante de esta viscosidad no es de aplicación para los motores de los vehículos participantes, ya que no se protege el motor de forma adecuada; en el caso del 38,09 \% de los vehículos participantes que utilizan un aceite 10W30, se demuestra que la viscosidad se mantiene, ya que el rango de protección y lubricación óptima es de $92^{\circ} \mathrm{C}$ a $107^{\circ} \mathrm{C}$, por tal razón este lubricante estará menos expuesto al efecto de cizallamiento en las zonas de mayor presión y temperatura del motor. En el proyecto la mayoría de los vehículos poseen motores que operan a estas temperaturas estableciendo que un aceite 10W30 es el indicado para aprovechar la vida útil del lubricante y proteger el motor de forma adecuada.

Luego del estudio e interpretación técnica de los análisis químicos, se determina que el lubricante puede durar hasta $7.000 \mathrm{~km}$ de recorrido (en condiciones severas de operación) sin comprometer la integridad y la vida útil del motor, utilizando un lubricante de base mineral debidamente certificado, pudiendo extenderse aún más este periodo al aplicar un mantenimiento y mayor seguimiento técnico.

Respecto a la contaminación ambiental:

\begin{tabular}{|c|c|c|c|}
\hline \multicolumn{4}{|c|}{ Generación de lubricante usado de motor por los taxis en la ciudad de Cuenca } \\
\hline Taxis & $\begin{array}{c}\text { Periodo de cambio de acei- } \\
\text { te (km recorrido/semanas) }\end{array}$ & $\begin{array}{c}\text { Número de cambios } \\
\text { de aceite al año }\end{array}$ & $\begin{array}{c}\text { Total galones lubri- } \\
\text { cante usado al año }\end{array}$ \\
\hline 2.614 & $3.500 / 3$ & 17 & $45.300,6$ \\
\hline Proyección de generación de lubricante usado de motor por los taxistas en la ciudad de Cuenca
\end{tabular}

Tabla 1. Generación de lubricante de motor usado de la población en estudio a periodos normal y extendido. Fuente: Elaboración propia a partir de los datos de la UMT y resultados obtenidos.

Con los resultados obtenidos durante el estudio (Tabla 1), demostramos que al realizar los cambios de aceite del motor cada $7.000 \mathrm{~km}$ de recorrido se reduciría en un 50\% la generación de desechos contaminantes del parque automotor de taxis en la ciudad de Cuenca, esto constituiría un gran avance en la conser- vación del medio ambiente, pudiendo ser proyectado a nivel nacional e internacional.

Respecto a los costos de mantenimiento por cambio de aceite y filtro del motor, al realizarlos a los $7.000 \mathrm{~km}$ de recorrido, se reducirían en un $50 \%$. \|\|$\|$ 


\section{Referencias bibliográficas}

[1] Analsyst, "Physical Properties tests", diciembre de 2010, http://www.analystsinc.com/pages/3437/

[2] Analsyst, "Spectrochemical analysis", diciembre de 2010, http://www.analystsinc.com/pages/3436/

[3] Deutsche Gesellschaft für Technische Zusammenarbeit (GTZ), "tecnología del automóvil" Tomo 2, 20a edición alemana-Editorial Revetré, S.A., pp. 545-446.

[4] Enrique Celis, "Aceite uso y propiedades", agosto de 2010,

[5] ETAPA (Empresa Pública Municipal de Telecomunicaciones, Agua Potable, Alcantarillado y Saneamiento de Cuenca), "Reciclado de aceites lubricantes en la ciudad de Cuenca", Abril 2010, http://www.etapa.net.ec/DGA/dga_pro_rec_ace_ que_hac.aspx

[6] Jaramillo, Fulvio, redrován, Fernando y Urgilés, Diego, "Vida Útil de los Lubricantes según sus fabricantes, Vida Útil del lubricante basado en análisis químicos de los mismos, Vida Útil del lu- bricante según los empíricos del medio", Trabajo de investigación, Cuenca, Noviembre de 2008, pp. 3-75.

[7] Los autores, "Recopilación de la información de las encuestas realizadas", Cuenca, Junio de 2010, pp. 18-27.

[8] Luna, Raúl, "Inyección a Gasolina", Cuenca, Marzo de 2009.

[9] Luna, Raúl, "Motores de Combustión Interna 1", Cuenca, Septiembre de 2008.

[10] Narváez, Paúl, "Cap. Motores de Combustión Interna 1", Cuenca, Septiembre de 2008.

[11] UMT (Unidad Municipal de Tránsito), "Registro de unidades de servicio de transporte de taxi", Cuenca, abril de 2010.

[12] Walpole, Ronald; MYERS, Raymond, "Probabilidad y Estadística para Ingenieros”, Sexta Edición, Editorial Prentice-Hall, México, 1999.

[13] Widman International, "Análisis de aceite", diciembre de 2010, http://www.widman.biz/ 